\title{
Juvenile systemic sclerosis - observations of one clinical centre
}

\author{
Lidia Rutkowska-Sak ID, Piotr Gietka ID , Agnieszka Gazda, Beata Kołodziejczyk
}

Clinic of Developmental-Age Rheumatology, National Institute of Geriatrics, Rheumatology, and Rehabilitation, Warsaw, Poland

\begin{abstract}
Objectives: The systemic form of scleroderma (SSc) in children is a very rare disease; therefore, it is recognized relatively late, which increases the risk of complications. The aim of the study was to assess the clinical symptoms of juvenile systemic sclerosis (JSSc) in our cohort patients.

Material and methods: A group of $(N=22)$ scleroderma patients aged between 2 and 16 years were observed. Demographic data and all clinical results obtained during 16 years of observation in the clinic of rheumatic diseases of developmental age were collected and analysed.

Results: In all observed children the major JSSc criterion was found, i.e. skin thickening proximal to the metacarpal phalangeal and/or metatarsophalangeal joints. Other symptoms are presented as follows: nailfold capillary abnormalities $-100 \%$, Raynaud's phenomenon $-90.9 \%$, sclerodactyly $-27.3 \%$, digital tip ulcers $-27.3 \%$, dysphagia $-18.2 \%$, gastroesophageal reflux $-27.3 \%$ (assessed in only 10 children), arrhythmias $-22.7 \%$, heart failure $-9.1 \%$, new-onset arterial hypertension $-9.1 \%$, pulmonary fibrosis $-72.7 \%$, pulmonary arterial hypertension $-9.1 \%$, neuropathy $-13.6 \%$, carpal tunnel syndrome $-4.5 \%$, tendon friction rubs $-4.5 \%$, arthritis $-22.7 \%$, and myositis $-13.6 \%$. There were no cases of renal crisis. Decreased diffusing capacity of oxygen was confirmed in 12 patients (58.3\%). The presence of antinuclear antibodies was noticed in $86.7 \%$ of patients, and among SSc selective autoantibodies: anticentromere $-31.8 \%$, anti-topoisomerase I-18.2\%, anti-PM-SCl 100 or $75-45.5 \%$, anti-RP11, Th/To, PCNA in total in $27.3 \%$ were presented. In $4.5 \%$ of cases, apart from the presence of anti-PM-Scl autoantibodies, positive lupus band test, reduced concentration of complement, and antiphospholipid antibodies were also found. In 59\% of studied children, the body mass index was below the 25 th percentile.

Conclusions: The presented retrospective analysis shows that the occurrence of Raynaud's phenomenon with changes in nailfold capillaroscopy is the best screening toll for the assessment of risk of JSSc. All patients of developmental age with Raynaud's phenomenon, especially in the case of the appearance of antinuclear antibodies, should be monitored with capillaroscopy regardless of other laboratory or imaging tests.
\end{abstract}

Key words: juvenile systemic sclerosis, clinical picture, Raynaud's phenomenon, capillaroscopy.

\section{Introduction}

Juvenile systemic sclerosis (JSSC) is considered to be one of the most severe inflammatory diseases of connective tissue. It is characterized by chronically progressive fibrosis of tissues and organs.

In developmental age, before the age of 16 years, diagnosis of JSSc is very rare. Its incidence at this age is estimated at 0.27 cases per million. According to statistics, children under 10 years of age account for about $3 \%$ of all the systemic scleroderma (SSc) cases in children and adults, while patients between 10 and 20 years of age account for $1.2-9 \%$ of all cases [1, 2].

The aetiology of JSSc remains unknown. Some studies show that the disease is more common in subjects with a certain genetic predisposition, such as the presence of the HLA-DRB1*10 antigen [3], or after exposure to specific environmental factors (various viruses, toxins, drugs). The more frequent occurrence in girls after

\section{Address for correspondence:}

Lidia Rutkowska-Sak, Clinic of Developmental-Age Rheumatology, National Institute of Geriatrics, Rheumatology and Rehabilitation, 1 Spartanska St., 02-637 Warsaw, Poland, e-mail: lidia.rutkowska-sak@spartanska.pl

Submitted: 01.09.2021; Accepted: 06.12.2021 
Table I. Classification of juvenile systemic sclerosis [6]

\begin{tabular}{c} 
Diffuse juvenile systemic sclerosis \\
\hline $\begin{array}{c}\text { Limited juvenile systemic sclerosis, formerly known } \\
\text { as CREST syndrome }\end{array}$ \\
\hline $\begin{array}{c}\text { Overlap syndrome juvenile systemic sclerosis, } \\
\text { e.g. scleromyositis, less often with systemic lupus } \\
\text { erythematosus, etc. }\end{array}$ \\
\hline
\end{tabular}

CREST syndrome - the name comes from the first letter of the main symptoms of the disease: Calcinosis, Raynaud's Phenomenon, Esophageal dysfunction, Sclerodactyly, Telangiectasia.

8 years of age may point to hormonal predisposition, and low birthweight is considered as an independent risk factor of SSc $[4,5]$.

In the pathogenesis of the disease, the involvement of innate and adaptive immune responses with the production of autoantibodies is emphasized. Monocytes, fibrocytes, growth factors, and cytokines are involved in the progression of the disease. Early changes in microvasculature with endothelial cell dysfunction and activation of mechanisms leading to their transformation into myofibroblasts are observed. Chronic and progressive fibrosis and ischaemia of skin tissues and internal organs causes their irreversible damage.

In 2007, the $3^{\text {rd }}$ International Rheumatological Societies - Paediatric Rheumatology European Society (PRES)/ American College of Rheumatology (ACR)/European League Against Rheumatism (EULAR) defined provisional classification criteria for the diagnosis of JSSc. Their sensitivity is $90 \%$ and specificity is $96 \%$ (Table I) [6].

Despite the passage of many years, they still constitute the basis for the diagnosis of JSSc (Table II), although nowadays the classification criteria for systemic sclerosis for adults, established by ACR/EULAR in 2013, may be a guide for analysis of the diagnosis and course of the disease in children [7] (Table I, II).

Diffuse cutaneous SSc is more commonly diagnosed in children than limited systemic sclerosis, which more often is diagnosed in adults, and the prognosis in SSc is better in children than in adults [8].

In recent years, it has been proposed that, in the course of JSSC, tools (indicators) to assess the severity of the disease should be used, i.e. the Juvenile Systemic Sclerosis Severity Score (J4S) [9]. The severity of the disease is defined on a 5 -point scale, ranging from inactivity, through mild, moderate, and severe, to critical. Disease severity assessment involves a clinical analysis of 9 organ systems/organs, with a maximum score of 2 to 8 points (points), based on their clinical relevance. Organ changes are precisely defined for each degree of activity. Hence, the greatest importance is assigned to the involvement of the lungs and heart (8 points each), less to the involvement of the kidneys, gastrointestinal tract, blood vessels, skin, and general symptoms
Table II. The Paediatric Rheumatology European Society/ American College of Rheumatology/European League Against Rheumatism provisional classification criteria for juvenile systemic sclerosis [7]

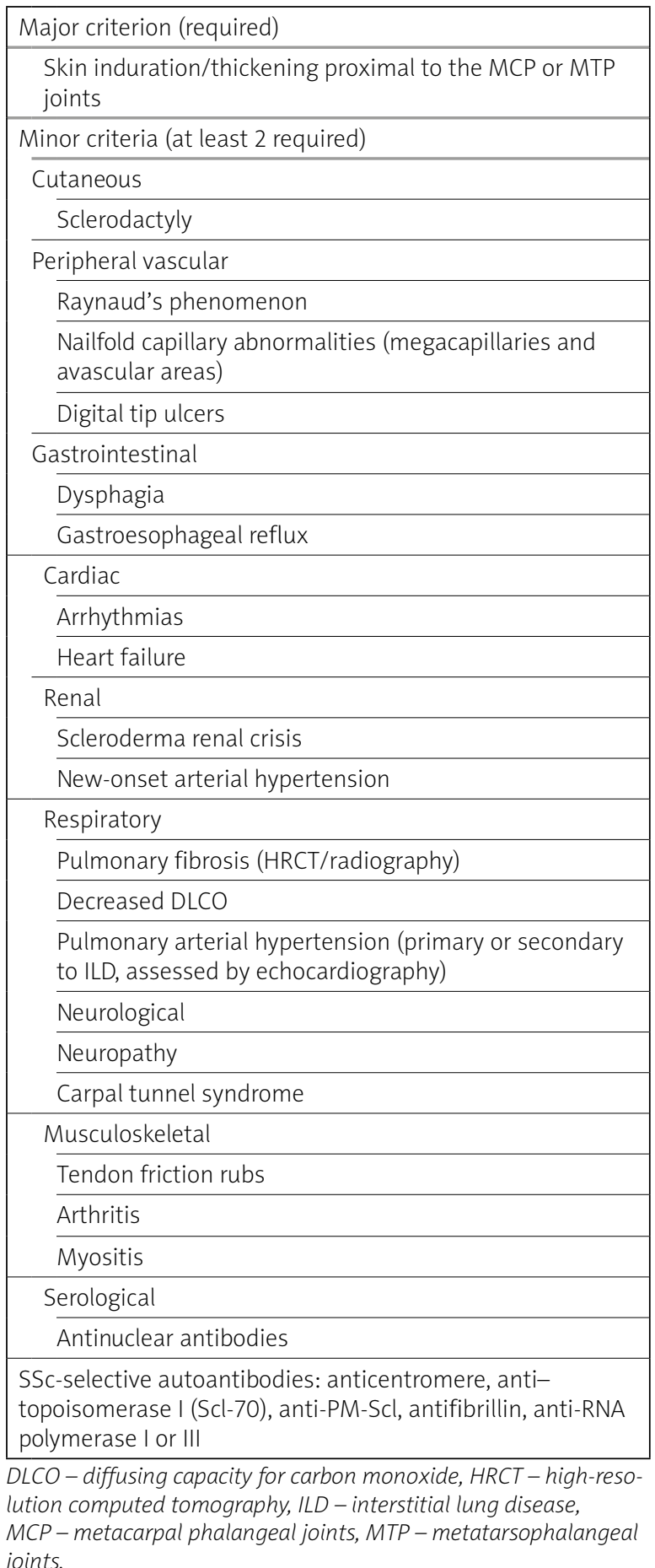

(4 points each), and the lowest to the involvement of the osteoarticular system and muscles (2 points each) [9].

The International Inceptions Cohort for JSSC is still working on validating the criteria for systemic sclerosis 
activity in adult patients for patients of developmenta age. The tools such as the Valentini disease activity index (VDAi), revised EUSTAR index (rEUSTARi), and physician's global assessment (PGA) seem to show good validity with the modified Rodnan skin score (mRSS) [10]. Authors concluded that the above-mentioned measurement tools may also be acceptable for the measurement of disease activity in paediatric SSc patients.

\section{Material and methods}

The aim of this study was to present the clinical picture of patients with JSSc hospitalized in the clinic of rheumatology of developmental age, who had been observed for the last 16 years. In most cases, the children had been treated in another rheumatological or paediatric hospital previously, sometimes for several years, or the disease was not diagnosed in their place of residence for a long time. The study was a retrospective analysis based on clinical documentation and is part of our scientific statutory work.

The group of observed patients included in total $n=22$ patients: $n=15(68.2 \%)$ females and $n=6(31.8 \%)$ males; aged from 6 to 16 years (mean 11.09 years) and one 2-year-old girl. The studied children were at very different stages of the disease. The duration of the disease ranged from 1 to 10 years (mean 4.09 years); in 9 children the disease has been present for over 5 years. Based on the medical history, the disease started in their cases between the $1^{\text {st }}$ and the $12^{\text {th }}$ year of life (on average at the age of 6.8 years). All children met the 2007 JSSc classification criteria [6].

\section{Results}

According to the classification criteria for the diagnosis of JSSc, in all (100\%) children the major JSSc criterion was found, i.e. skin induration/thickening proximal to the metacarpal phalangeal and/or metatarsophalangeal joints [6]. In the majority of patients (69\%) skin thickening affected other areas of the body, including the neck, trunk, buttocks, thighs, and limbs in the area of shoulder, elbow, wrist, hip, and knee joints. In 59\% of patients the distinct feature was poor facial expressions with smoothing of the skin of the cheek folds, narrow red of the lips, and a translucent mesh of blood vessels.

The percentage of children with changes in individual categories included in the minor JSSC criteria are presented in Table III.

Noteworthy features of restriction not included in the clinical criteria but observed in 12 children undergoing spirometry and body plethysmography included: acroosteolysis in 50\% of all patients, osteoporosis in $63.6 \%$, muscle weakness in $31.8 \%$, and heart valve de- fects in $22.7 \%$ of children. Rheumatoid factor was found in $22.7 \%$ of patients, and a similar percentage of patients had proteinuria. In $59 \%$ of the children, the body mass index (BMI) was below the $25^{\text {th }}$ percentile. Although recently mRSS has been generally accepted as a method of clinical assessment of the extent of skin lesions in patients with SSc in adults as in children, as is reported in this retrospective analysis, it had been used only in 5 of our patients, and values ranged from 18 to 40/51.

Comparing the observations in children with a disease onset of less than 2 years before admission to the clinic $(n=8)$ with the observations in children whose disease duration was longer, the least noticeable differences were in Raynaud's phenomenon - only $13.6 \%$ of children with shorter duration of the disease had less severe symptoms. All other disease symptoms were more severe, depending on the duration of the disease, even by more than $40 \%$. Spirometry and gastroscopy were not assessed because these routine examinations were performed only in children with longer duration of the disease.

Psychological assessment revealed low mood in 90\% of children with a longer duration of SSc, and depressive state was confirmed in $20 \%$ of them.

In patients with the onset of the disease over 7 years of age sclerodactyly and acroosteolysis as well as the presence of Scl-70 autoantibodies were found more often than others. Also, younger children met, on average, $5 \mathrm{mi}$ nor criteria, while older ones ( $>7$ y.o.) met 10 on average. Ultimately, $54.6 \%(n=12)$ patients were qualified as diffuse JSSC (DJSSC), 13.6\% $(n=3)$ were classified as limited subtype JSSC (LJSSC), and 31.8\% $(n=7)$ were diagnosed with overlap syndrome JSSc (OvJSSc). Among patients with LJSSc, the symptoms from the osteoarticular system were less pronounced, and in the group of children with OvJSSC, changes in internal organs were not expressed by strong symptoms. In the OvJSSc group $n=6$ patients were classified as scleromyositis (SSC-PM) with weakened muscle strength and a slight increase in muscle enzyme levels, and in 3 cases muscle biopsy confirmed myositis and they also had calcareous foci. Finally, 1 patient from the OvJSSc group met the criteria for systemic lupus erythematosus in addition to the features of SSc.

Patients had been treated with glucocorticosteroids, intravenous and oral cyclophosphamide, methotrexate, azathioprine, intravenous infusions of immunoglobulins, mycophenolate mofetil, and therapies targeting organ complications.

\section{Discussion}

The symptoms we analysed on the basis of the medical history of a small group of patients with JSSC at different stages of their disease cannot be easily compared to analyses of large cohorts of patients, especially 
Table III. Clinical characteristics of the patients according to the Paediatric Rheumatology European Society/ American College of Rheumatology/European League against Rheumatism provisional classification criteria for juvenile systemic sclerosis

\begin{tabular}{|c|c|c|}
\hline \multicolumn{3}{|l|}{ Major criterion } \\
\hline \multicolumn{2}{|c|}{ Skin induration/thickening proximal to the MCP or MTP joints } & $100 \%$ \\
\hline \multicolumn{3}{|l|}{ Minor criteria } \\
\hline Cutaneous & Sclerodactyly & $27.3 \%$ \\
\hline \multirow[t]{3}{*}{ Peripheral vascular } & Raynaud's phenomenon & $90.9 \%$ \\
\hline & Nailfold capillary abnormalities (megacapillaries and avascular areas) & $100 \%$ \\
\hline & Digital tip ulcers & $27.3 \%$ \\
\hline \multirow[t]{2}{*}{ Gastrointestinal } & Dysphagia & $18.2 \%$ \\
\hline & Gastroesophageal reflux & $27.3 \%$ \\
\hline \multirow[t]{3}{*}{ Cardiac } & Arrhythmias & $22.7 \%$ \\
\hline & Heart attack & 0 \\
\hline & Heart failure & $9.1 \%$ \\
\hline \multirow[t]{2}{*}{ Renal } & Scleroderma renal crisis & 0 \\
\hline & New-onset arterial hypertension & $9.1 \%$ \\
\hline \multirow[t]{3}{*}{ Respiratory } & Pulmonary fibrosis (HRCT/radiography) & $72.7 \%$ \\
\hline & Decreased DLCO & $58.3 \%$ \\
\hline & Pulmonary arterial hypertension (assessed by echocardiography) & $9.1 \%$ \\
\hline \multirow[t]{2}{*}{ Neurological } & Neuropathy & $13.6 \%$ \\
\hline & Carpal tunnel syndrome & $4.5 \%$ \\
\hline \multirow[t]{3}{*}{ Musculoskeletal } & Tendon friction rubs & $4.5 \%$ \\
\hline & Arthritis & $22.7 \%$ \\
\hline & Myositis & $13.6 \%$ \\
\hline \multirow[t]{5}{*}{ Serological } & Antinuclear antibodies & $86.7 \%$ \\
\hline & Anticentromere antibodies & $31.8 \%$ \\
\hline & Anti-topoisomerase I antibodies & $18.2 \%$ \\
\hline & PM-Scl antibodies & $45.5 \%$ \\
\hline & Anti-RNA polymerase III antibodies & $9.09 \%$ \\
\hline \multicolumn{3}{|c|}{ Symptoms/test results not included in the criteria } \\
\hline $\begin{array}{l}\text { Restriction in spirometry tests } \\
\text { (assessed in children) }\end{array}$ & \multicolumn{2}{|l|}{$12 / 12$} \\
\hline BMI below the $25^{\text {th }}$ percentile & \multicolumn{2}{|l|}{$59 \%$} \\
\hline Acroosteolysis in radiography & \multicolumn{2}{|l|}{$50 \%$} \\
\hline The presence of RF & \multicolumn{2}{|l|}{$22.7 \%$} \\
\hline Proteinuria & \multicolumn{2}{|l|}{$22.7 \%$} \\
\hline Osteoporosis & \multicolumn{2}{|l|}{$63.6 \%$} \\
\hline Muscle weakness & \multicolumn{2}{|l|}{$31.8 \%$} \\
\hline Heart valve defects & \multicolumn{2}{|l|}{$22.7 \%$} \\
\hline
\end{tabular}

$B M I$ - body mass index, DLCO - diffusing capacity for carbon monoxide, HRCT - high-resolution computed tomography, MCP - metacarpal phalangeal joints, MTP - metatarsophalangeal joints, RF - rheumatoid factor.

in prospective studies. The onset of the disease in the described patients occurred on average at the age of 6.8 years. In other authors this borderline varies [11-13].
However, many observations are similar - girls are suffer more often from JSSC than boys and the leading symptom of the onset of the disease is RP [11-13]. According 
to Scalapino [11] RP occurs in 74-100\%, according to Androvic [12] in 93\%, and according to Foeldvari et al. [13] in $91 \%$ of patients. Different images of the nailfold capillaries, were in all our patients and worsening with the duration of the disease, were also observed by Stevens et al. [14] in $92 \%$ of $64 \mathrm{JSSC}$ patients included in this study.

In the presented study we found interstitial lung infiltrates in $72.7 \%$ of children; in 2006 Martini et al. [15] found these changes in a much smaller number of patients, but it can be explained that it was the beginning of the disease in comparison with our patients. Borowiec et al. [16] found interstitial lung disease lesions in $86.7 \%$ of JSSc patients. The incidence of asymptomatic cardiac arrhythmias in the described patients was observed in 4 cases as well as heart failure symptoms, and it was similar to late observations by Borowiec et al. [16]. Gastroesophageal reflux was observed in $27.3 \%$ of children, and dysphagia in $18.2 \%$.

Of note, our older observations from years 1992 and 1995 presented among 11 observed patients with JSSC, 4 of them had cardiac or pyloric atony, and 5 had gastroesophageal reflux $[17,18]$. In the current observations, distortion of the gallbladder was evident in 7 children, cholestasis in 9 children - regardless of the time and activity of the disease - and intrahepatic cholestasis was observed in 4 children. Among 5 children who died in the course of JSSC, 4 had numerous erosions and ulcers in the gastrointestinal tract, and 3 had fibrotic streaks. The lesions found in the digestive tract are undoubtedly one of the factors contributing to the reduction of $\mathrm{BMI}$ in $59 \%$ of the children with JSSc we currently observe. Stevens et al. [14] found that changes in the digestive tract in children with JSSC is the factor that most reduces the quality of life of these patients, more than advanced skin lesions.

Skin lesions affecting the face are especially difficult to accept for patients with JSSc. Due to facial lesions, SSc is known as the disease that "steals the face".

Muscle weakness and features of cardiovascular dysfunction weakened the physical functioning of the observed children, and it is known that the mental functioning of a child correlates mainly with its physical functioning, hence the low mood and depressive states were observed frequently in our patients [19]. In the presented group were also patients with features of other rheumatic diseases (overlap JSSc-OvJSSc).

In 2021 the results from a large cohort international JSSc study were published and showed significant differences between patients with JSSC and overlap features [20]. The authors concluded that patients with overlap symptoms more often had interstitial lung disease and higher disease activity score in physicians assessment; this is an indication that this group of patients should not be treated as mildly suffering from scleroderma.

To sum up, a very severe orphan connective tissue disease in children such as JSSc is often diagnosed very late. Generalized, life-threatening symptoms of the disease sometimes may develop quickly. The disease itself is mentally poorly accepted by the patients. Our findings are similar to those from other investigators and clinicians, and pointed to isolated Raynaud's phenomenon along with positive antinuclear antibody as well as nailfold capillary changes as features of the usual insidious onset of the disease [21].

\section{Study limitations}

The presented work has several limitations: first of all, its retrospective nature does not allow for modification of the procedure and supplementation of data. Secondly, children came to the clinic at various stages of the disease, which made comparisons difficult. Age differences (from 2 to 16 years of age) may also influence the differences in the clinical picture of the disease. The relatively small group of patients $(n=22)$ is due to the rarity of this disease in developmental age and should not be a clear limitation of the assessment of patients.

\section{Conclusions}

The occurrence of Raynaud's phenomenon, although often observed in potentially healthy children, is associated with a high risk of the development of JSSC and requires monitoring, especially in the case of the serum presence of antinuclear antibodies. All patients with this phenomenon should be monitored, and nailfold capillaroscopy should be performed periodically as a screening tool.

The authors declare no conflict of interest.

\section{References}

1. Foeldvari I, Klotsche J, Torok KS, et al. Are diffuse and limited juvenile systemic sclerosis different in clinical presentation? Clinical characteristics of a juvenile systemic sclerosis cohort. J Scleroderma Related Dis (JSRD) 2019; 4: 49-61, DOI: 10.1177/2397198318790494.

2. Royle JG, Lanyon PC, Grainge MJ, et al. The incidence, prevalence, and survival of systemic sclerosis in the UK Clinical Practice Research Datalink. Clin Rheumatol 2018; 37: 2103-2111, DOI: 10.1007/s10067-018-4182-3.

3. Stevens AM, Kanaan SB, Torok KS, et al. Brief report: HLADRB1, DQA1, and DQB1 in juvenile-onset systemic sclerosis. Arthritis Rheum 2016; 68: 2772-2777, DOI: 10.1002/ art.39765. 2772. 
4. Abbot S, Bossingham D, Proudman S, et al. Risk factors for the development of systemic sclerosis: a systematic review of the literature. Rheumatol Adv Pract 2018; 2: rky041, DOI:10.1093/ rap/rky041.

5. Donzelli G, Carnesecchi G, Amador C, et al. Fetal programming and systemic sclerosis. Am J Obstet Gynecol 2015; 213: 839. e1-8, DOI: 10.1016/j.ajog.2015.07.034.

6. Zulian F, Woo P, Athreya BH, et al. The Pediatric Rheumatology European Society/American College of Rheumatology/European League against Rheumatism provisional classification criteria for juvenile systemic sclerosis. Arthritis Rheum 2007; 57: 203-212, DOI: 10.1002/art.22551.

7. Van den Hoogen F, Khanna D, Fransen J, et al. Classification Criteria for Systemic Sclerosis: an ACR-EULAR Collaborative Initiative. Arthritis Rheum 2013; 65: 2737-2747, DOI: 10.1002/ art.38098.

8. Foeldvari I. Systemic sclerosis in childhood. Rheumatology (Oxford) 2006; 45 Suppl 3: iii28-iii29, DOI:10.1093/rheumatology/ kel292.

9. La Torre F, Martini G, Russo R, et al. A preliminary disease severity score for juvenile systemic sclerosis. Arthritis Rheum 2012; 64: 4143-4150, DOI: 10.1002/art.34652

10. Klotsche J, Foeldvari I, Kasapçopur Ö, et al. Validation of disease activity measures in patients withJSSC. J Scleroderma Related Dis 2020; 5 (1_suppl): 1-307, DOI: 10.1177/2397198319898367.

11. Scalapino K, Arkachaisri T, Lucas M, et al. Childhood onset systemic sclerosis: classification, clinical and serologic features, and survival in comparison with adult onset disease. J Rheumatol 2006; 33: 1004-1013.

12. Adrovic A, Şahin S, Barut K, Kasapçopur Ö. Juvenile scleroderma: a referral center experience. Arch Reumatol 2018; 33 344-351, DOI:10.5606/ArchRheumatol.2018.6578.

13. Foeldvari I, Zvahania M, Birdi N, et al. Favourable outcome in 135 children with juvenile systemic sclerosis: results of a mult
- national survey. Rheumatology (Oxford) 2000; 39: 556-559, DOI: 10.1093/rheumatology/39.5.556.

14. Stevens BE, Torok KS, Li SC, et al. Clinical characteristics and factors associated with disability and impaired quality of life in children with juvenile systemic sclerosis. Arthritis Care Res (Hoboken) 2018; 70: 1806-1813, DOI: 10.1002/acr.23547.

15. Martini G, Foeldvari I, Russo R, et al. Juvenile Scleroderma Working Group of the Pediatric Rheumatology European Society. Systemic sclerosis in childhood: clinical and immunologic features of 153 patients in an international database. Arthritis Rheum 2006; 54: 3971-3978, DOI: 10.1002/art.22207.

16. Borowiec A, Dąbrowski R, Woźniak J, et al. Cardiovascular assessment of asymptomatic patients with juvenile-onset localized and systemic scleroderma: 10 years prospective observation. Scan J Rheumatol 2012; 41: 33-38, DOI: 10.3109/03009742.2011.609489.

17. Rutkowska-Sak L. Obraz dróg żółciowych u dzieci chorych na zapalne układowe choroby tkanki łącznej. Ped Pol 1992; 67: 660-666.

18 Rutkowska-Sak L, Legatowicz-Koprowska M, Ryżko J, Socha J. Zmiany w układzie pokarmowym u dzieci chorych na zapalne układowe choroby tkanki łącznej. Ped Pol 1995; 70: 235-241.

19. Colasanto M, Madigan S, Korczak DJ. Depression and inflammation among children and adolescents: a meta-analysis. J Affect Disord 2020; 277: 940-948, DOI: 10.1016/j.jad.2020.09.025.

20. Foeldvari I, Klotsche J, Kasapcopur O, et al. Pos1304 Juvenile systemic sclerosis (JSSC) patients with overlap characteristics do not have mild disease. Results from the jssc inception cohort. www.juvenilescleroderma.com. Ann Rheum Dis 2021; 80: 934.

21. Stevens AM, Torok KS, Li SC, et al. Immunopathogenesis of juvenile systemic sclerosis. Front Immunol 2019; 10: 1352, DOI:10.3389/fimmu.2019.01352. 